\title{
Dichotomizing Level of Pial Collaterals on Multiphase CT Angiography for Endovascular Treatment in Acute Ischemic Stroke: Should It Be Refined for 6-Hour Time Window?
}

\author{
Ho Geol Woo, $\mathrm{MD}^{1}$, Cheolkyu Jung, $\mathrm{MD}, \mathrm{PhD}^{2}$, Leonard Sunwoo, $\mathrm{MD}$, $\mathrm{PhD}^{2}$, Yun Jung Bae, MD, $\mathrm{PhD}^{2}$, \\ Byung Se Choi, MD, PhD ${ }^{2}$, Jae Hyoung Kim, MD, $\mathrm{PhD}^{2}$, Beom Joon Kim, MD, PhD ${ }^{3}$, \\ Moon-Ku Han, MD, PhD ${ }^{3}$, Hee-Joon Bae, MD, PhD 3 , Seunguk Jung, $\mathrm{MD}^{4}$, Sang-Hoon Cha, MD, PhD \\ ${ }^{1}$ Department of Neurology, Ewha Womans University College of Medicine, Seoul, Korea \\ ${ }^{2}$ Department of Radiology, Seoul National University Bundang Hospital, Korea \\ ${ }^{3}$ Department of Neurology, Seoul National University Bundang Hospital, Korea \\ ${ }^{4}$ Department of Neurology, Gyeongsang National University Changwon Hospital, Changwon, Korea \\ ${ }^{5}$ Department of Radiology, Chungbuk National University College of Medicine, Cheongju, Korea
}

Purpose: Although endovascular treatment is currently thought to only be suitable for patients who have pial arterial filling scores $>3$ as determined by multiphase computed tomography angiography (mpCTA), a cut-off score of 3 was determined by a study, including patients within 12 hours after symptom onset. We aimed to investigate whether a cut-off score of 3 for endovascular treatment within 6 hours of symptom onset is an appropriate predictor of good functional outcome at 3 months.

Materials and Methods: From April 2015 to January 2016, acute ischemic stroke patients treated with mechanical thrombectomy within 6 hours of symptom onset were enrolled into this study. Pial arterial filling scores were semi-quantitatively assessed using mpCTA, and clinical and radiological parameters were compared between patients with favorable and unfavorable outcomes. Multivariate logistic regression analysis was then performed to investigate the independent association between clinical outcome and pial collateral score, with the predictive power of the latter assessed using C-statistics.

Results: Of the 38 patients enrolled, 20 (52.6\%) had a favorable outcome and 18 had an unfavorable outcome, with the latter group showing a lower mean pial arterial filling score $(3.6 \pm 0.8$ vs. $2.4 \pm 1.2, P=0.002$ ). After adjusting for variables with a P-value of $<0.1$ in univariate analysis (i.e., age and National Institutes of Health Stroke Scale score at admission), pial arterial filling scores higher than a cut-off of 2 were found to be independently associated with favorable clinical outcomes ( $P=0.012)$. C-statistic analysis confirmed that our model had the highest prediction power when pial arterial filling scores were dichotomized at $>2$ vs. $\leq 2$.

Conclusion: A pial arterial filling cut-off score of 2 as determined by mpCTA appears to be more suitable for predicting clinical outcomes following endovascular treatment within 6 hours of symptom onset than the cut-off of 3 that had been previously suggested.

Key Words: Stroke; Computed tomography angiography; Thrombectomy

\section{Correspondence to:}

Cheolkyu Jung, MD, PhD

Department of Radiology, Seoul

National University Bundang Hospital, 82 Gumi-ro 173beon-gil, Seongnam

13620, Korea

Tel: +82-31-787-7623

Fax: +82-31-787-4011

E-mail: jck0097@gmail.com

Received: March 18, 2019

Revised: May 13, 2019

Accepted: June 9, 2019 Copyright $\odot 2019$ Korean Society of
Interventional Neuroradiology
This is an Open Access article distributed under the terms of
the Creative Commons Attribution Non-Commercial License
(http://creativecommons.org/licenses/by-nc/3.0) which
permits unrestricted non-commercial use, distribution, and
reproduction in any medium, provided the original work is
properly cited.
pISSN 2093-9043
eISSN 2233-6273 


\section{INTRODUCTION}

Endovascular treatment in patients with acute ischemic stroke has evolved substantially in recent years. Five randomized clinical trials have led to the establishment of endovascular treatment using stent retriever devices as the standard of care for patients with proximal anterior circulation occlusion. ${ }^{1-3}$

Some studies have shown that the degree of leptomeningeal or pial collateral circulation on single-phase computed tomography angiography (spCTA) is associated with clinical outcome, infarct volume, hemorrhage transformation risk, and recanalization rates in acute stroke patients $s^{4-6}$ and infarct volume and clinical outcome in patients undergoing thrombolysis. ${ }^{7}$ Moreover, collateral circulation on dynamic CTA predicts clinical outcome at 3 months. ${ }^{8}$ However, although spCTA and dynamic CTA are widely used for pial collateral status evaluation for acute stroke, spCTA lacks temporal resolution and may mislabel pial collateral status, and dynamic CTA needs postprocessing. An alternative is multiphase computed tomography angiography (mpCTA), which as a time-resolved technique can give clinicians more accurate information on the degree and extent of collaterals. Indeed, mpCTA shows better interrater reliability and is associated with better clinical outcomes than spCTA when used to assess acute stroke patients, can be quickly performed, and yields images that can be interpreted without specialist assistance. ${ }^{9,10}$

The degree of pial collateral circulation on mpCTA is measured by the 6-point pial arterial filling score, with patients scoring 0-3 not likely to benefit from recanalization with a proximal intracranial occlusion in the anterior circulation admitted within 12 hours after symptom onset. ${ }^{9}$ However, we thought a different decision strategy using collateral status is needed for patients with a proximal intracranial occlusion in the anterior circulation admitted within 6 hours after symptom onset. Therefore, we aimed to investigate whether the current pial arterial filling score dichotomization of $0-3$ vs. 4-5 is appropriate in triaging patients for endovascular treatment with acute ischemic stroke and large vessel occlusion in the anterior circulation who were admitted within 6 hours after symptom onset.

\section{MATERIALS AND METHODS}

The Seoul National University Bundang Hospital Institutional
Review Board (IRB No. B-1608-357-115) approved this retrospective study, and informed consent was waived.

\section{Patient recruitment}

This study was a retrospective analysis of data prospectively collected from acute ischemic stroke patients who presented at our institution within 6 hours of symptom onset from April 2015 to January 2016. Patients were included if they met the following inclusion criteria: 1) baseline imaging data, including non-contrast computed tomography and mpCTA prior to endovascular treatment, were available, 2) there was occlusion of the intracranial segment of the internal carotid artery (ICA) or middle cerebral artery (MCA) (M1 or M2 segment), and 3) they had a National Institutes of Health Stroke Scale (NIHSS) score of 5 or higher. We excluded patients with poor-quality mpCTA data that prevented the assessment of pial collateral circulation and those who were lost to follow-up.

\section{Imaging protocol}

All patients underwent a standard scanning protocol at presentation using a 256-slice multidetector CT scanner (Brilliance iCT 256; Philips Medical Systems, Cleveland, $\mathrm{OH}$, USA). The mpCTA scanning parameters used were $80 \mathrm{kVp}$, $175 \mathrm{mAs}$, and $128 \times 0.625 \mathrm{~mm}$ detector collimation. A total of $90 \mathrm{~mL}$ of contrast was injected for the scan at a rate of $4-5$ $\mathrm{mL} / \mathrm{sec}$, followed by a $30-\mathrm{mL}$ normal saline chase at a rate of $3 \mathrm{~mL} / \mathrm{sec}$.

The first phase of imaging involved an aortic arch-to-vertex CTA scan that was initiated by the bolus tracking method with a trigger level of 150 Hounsfield units at the aortic arch, minimum scan delay, and an average dose length product of 1,100 to $1,200 \mathrm{mGy} \cdot \mathrm{cm}$. This was acquired in less than 3 seconds. The second phase started after a delay of 4 seconds and was acquired in 3 seconds from the vertex to skull base. The third phase was initiated after another 4-second delay with scanning in the opposite direction from the skull base to vertex. The scan was performed in this direction for the third phase because our scanner is not able to be repositioned within the time limit of 7 seconds between phases. Images from the first phase (i.e., the aortic arch-tovertex scan) were then reconstructed as 1.5 -mm-thick axial sections. In addition, 24-mm-thick slab multi-planar reformations of axial first to third phase images from the skull base to vertex were performed to assess the degree of pial collateral filling. 
This mpCTA protocol does not require additional contrast medium, and there is minimal extra radiation (approximately $177 \mathrm{mGy} \cdot \mathrm{cm}$ per phase) as the second- and third-phase images are acquired from the skull base to vertex only. 11,12

\section{Image analysis}

Radiologic data were independently assessed by a neurologist (H.G.W.) and 2 neuroradiologists (C.J. and L.S.) who were blinded to patient clinical symptoms. Pial arterial filling score was measured in accordance with a previously published report. ${ }^{9}$ Although there was excellent interrater reliability between the two radiologists ( $n=30, k=0.81, P<0.001)$ in a previous report, interrater reliability in our study was moderate on first interpretation ( $n=38, k=0.46, P<0.001$ ). Hence, we needed to clarify the three determinants of pial arterial filling score, i.e., the degree of phase delay, prominence, and extent of pial collaterals. The degree of phase delay was defined as the difference in the phase of pial collateral filling between thick axial maximum intensity projections of the head in the index territory and that in the contralateral territory. ${ }^{12}$ Reductions in the prominence and extent of pial collaterals were defined as when the number of pial vessels in the index territory was smaller than that in the contralateral territory, with adjustment for different phases of collateral filling between the index and contralateral territories. Ischemic regions with no vessels were defined as regions where one third or more of the lesions in the index territory had no collateral filling in any phase (Table 1).

Table 1 and Fig. 1 show an example template for each score (i.e., 0-6) based on the three determinants above. Our three raters then revised their pial collateral assessments according to this template, with a consensus required in cases of disagreement.

\section{Procedures}

A neurointerventionalist (C.J.) performed intraarterial treatment (IAT) of all the cases on a biplane angiography machine (Integris Allura, Philips, the Netherlands). We used a 9 French balloon guiding catheter. In case of a tortuous cervical ICA, a second guiding catheter was used for the coaxial system. The primary device for IAT was a stent retriever (Solitaire FR; Covidien, Irvine, California; Trevo-XP; Stryker, Kalamazoo, MI, USA). Mechanical aspiration device, thrombolytics, or permanent stenting were also used at the discretion of operator. The reperfusion grading was measured by using modified treatment in cerebral infarction before and after IAT. ${ }^{13}$

\section{Statistical methods}

Patient demographics, as well as clinical and radiological findings, were compared between patients with favorable (modified Rankin Scale [mRS] at 3 months $\leq 2$ ) and unfavorable (mRS at 3 months $>2$ ) outcomes using the Pearson chisquare test, independent $t$ test, and Mann-Whitney $U$ test as appropriate. Univariate and multivariate logistic regression analyses were then performed to identify factors associated with a favorable functional outcome. Variables with a P-value of $\leq 0.1$ in univariate analysis were included in multivariate analyses. We then determined the area under the receiver-operating characteristic curve (AUC) to assess model fit and compared AUC values between pial arterial filling scores dichotomized at various cut-off scores (i.e., $>4$ vs. $\leq 4,>3$ vs. $\leq 3$, and $>2$ vs. $\leq 2$ ). Areas under the receiver operating characteristic curves for these 2 scoring systems were compared using DeLong's test. All statistical analyses were performed

Table 1. Pial arterial filling score used in the current study within the symptomatic ischemic territory compared with the asymptomatic contralateral hemisphere using multiphase $\mathrm{CT}$ angiography

\begin{tabular}{lll}
\hline Score & Phase difference in filling of pial collateral & Prominence and extent of pial collateral \\
\hline 5 & No & Same or increased \\
\hline 4 & One phase & Same \\
3 & Two phases & Same \\
3 & One phase & Reduced prominence or reduced extent \\
2 & Two phases & Reduced prominence or reduced extent \\
2 & One phase & No vessel with one third or more defect than normal extent \\
1 & Two or more phases & A few visible vessels with whole territory defect of occluded vessel \\
0 & Two or more phases & No visible vessel with whole territory defect of occluded vessel \\
\hline
\end{tabular}

$\mathrm{CT}$, computed tomography. 
using SPSS Statistics 22 (IBM, Armonk, NY, USA) and Stata 13.0 software (Stata Corporation, College Station, TX, USA). P values $<0.05$ were considered statistically significant. Results are expressed as mean \pm standard deviation where appropriate.

\section{RESULTS}

\section{Patient characteristics}

Of the 838 patients in our database with acute ischemic

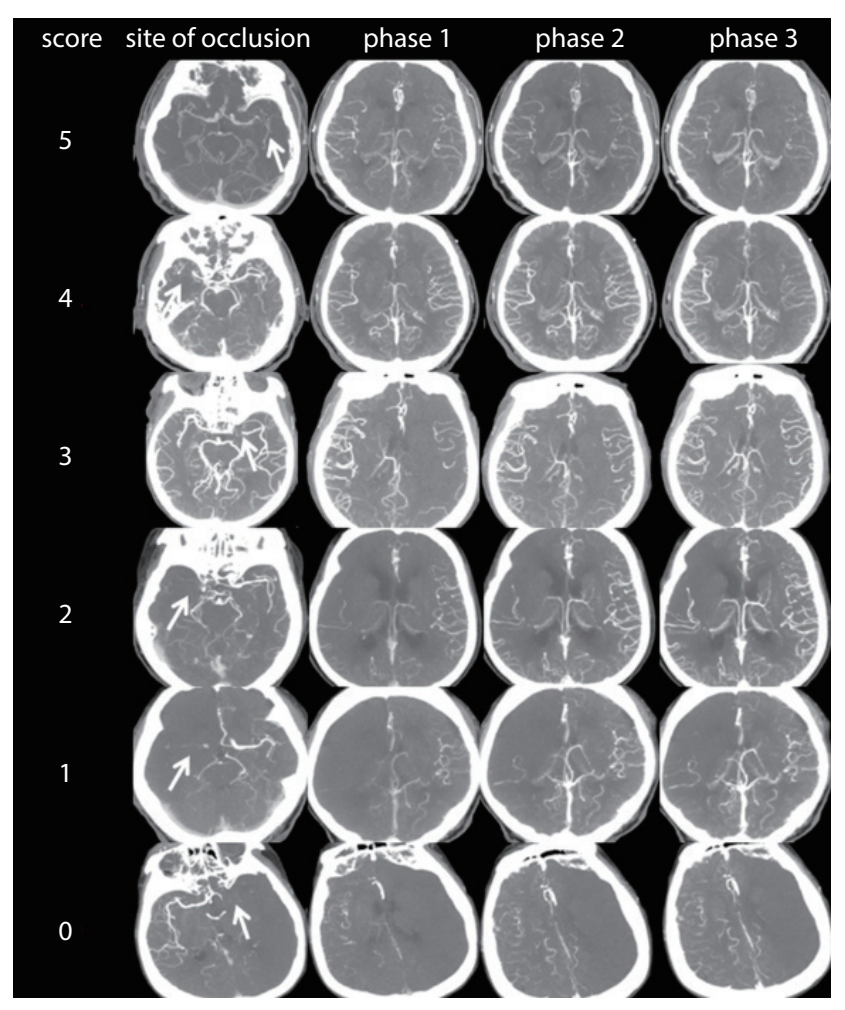

Fig. 1. Pial arterial filling score within the symptomatic ischemic territory using multiphase CT angiography images. Top row: images of a patient with a left MCA M1 segment occlusion (arrow) and no delay of phase in filling in of peripheral vessels, but with the same prominence and extent. Second row: images of a patient with a right MCA M1 segment occlusion (arrow) and a delay of 1 phase in filling in of peripheral vessels, but with the same prominence and extent. Third row: images of a patient with a left MCA M1 segment occlusion (arrow) and a delay of 2 phases in filling in of peripheral vessels, but with the same prominence and extent. Fourth row: images of a patient with a right MCA M1 segment occlusion (arrow) and a delay of 1 phase in filling in of peripheral vessels, but with decreased prominence and extent. Fifth row: images of a patient with a right MCA M1 segment occlusion (arrow) and just a few vessels visible in any phase within the occluded vascular territory. Bottom row: images of a patient with a left MCA M1 segment occlusion (arrow) and no vessels visible in any phase within the ischemic vascular territory. CT, computed tomograpy; MCA, middle cerebral artery. stroke, 257 patients underwent mpCTA within 6 hours of symptom onset. Among these, 208 patients who were not candidates for endovascular revascularization were excluded due to mild symptoms (NIHSS <4, 152 patients), no occlusion identified on mpCTA (46 patients), and hemorrhagic diathesis (10 patients). In total, 11 patients underwent endovascular treatment for occlusion in the basilar artery or vertebra artery, and 38 underwent endovascular treatment using a stent retriever for ICA/MCA occlusion identified on mpCTA. The mean age of enrolled patients was $72.4 \pm 10.2$ years, and $55.3 \%$ were male. Favorable outcomes were observed in 20 patients (52.6\%), whereas unfavorable outcomes were observed in 18 (47.4\%). The most common stroke subtype was cardioembolism (63.2\%). Thirty-one patients (81.6\%) were treated with intravenous tissue plasminogen activator before endovascular treatment, and 34 (89.5\%) had successful reperfusion. The mean pial arterial filling score was 3.0 \pm 1.2 .

\section{Interrater reliability of the pial arterial filling score}

Interrater reliability of the pial arterial filling score between a neurologist and two neuroradiologists, as assessed using unweighted $k$ statistics with 95\% confidence intervals (Cls), indicated substantial agreement $(n=38, k=0.64, P<0.001)$.

\section{Comparison of clinical and radiological parameters between patients with favorable and unfavorable outcomes}

Patient demographics and clinical and radiological parameters are shown in Table 2. Mean age was lower in the favorable (67.1 \pm 10.4 years) than in the unfavorable (78.3 \pm 6.0 years) outcome groups $(P<0.001)$, while pial arterial filling score was higher in the favorable (3.6 \pm 0.8$)$ than in the unfavorable (2.4 \pm 1.2$)$ outcome groups ( $P=0.002)$.

In our univariate analysis, younger age (odds ratio [OR], $0.825 ; 95 \% \mathrm{Cl}, 0.720-0.945 ; \mathrm{P}=0.006$ ) and lower NIHSS score at admission (OR, 0.839; 95\% Cl, 0.720-0.977; $\mathrm{P}=0.024)$ were significantly associated with favorable outcomes at 3 months. Higher pial arterial filling score (OR, 2.917; 95\% Cl, 0.321-6.443; $\mathrm{P}=0.008$ ) was also significantly associated with favorable outcomes. Because of the ambiguity of the score threshold for predicting clinical outcomes in endovascular treatment, we dichotomized the pial arterial filling score at $>4$ vs. $\leq 4$, $>3$ vs. $\leq 3$, and $>2$ vs. $\leq 2$ and used each dichotomization as a predictor variable in our analyses. Similar trends to those above were shown when the pial arterial filling score was dichotomized at $>2$ vs. $\leq 2$ (OR, 14.143; $\mathrm{P}=0.003)$. In contrast, 
pial arterial filling scores dichotomized at $>3$ vs. $\leq 3$ and $>4$ vs. $\leq 4$ were not significantly associated with clinical outcome. After adjusting for age and NIHSS score at admission, higher pial arterial filling scores and pial arterial filling scores dichotomized at $>2$ vs. $\leq 2$ were found to be independently associated with favorable clinical outcomes (Table 3). The pial arterial filling scores dichotomized at $>2$ vs. $\leq 2$ (AUC, 0.756; 95\% Cl, 0.621-0.89; $\mathrm{P}=0.249$ ) also effectively predicted favorable clinical outcomes at 3 months, with higher AUC than dichotomization at pial arterial filling scores dichotomized at $>3$ vs. $\leq 3$ and $>4$ vs. $\leq 4$ (AUC, $0.636 ; 95 \%$ Cl, 0.482-0.791;
$P=0.364$, and AUC, $0.550 ; 95 \% \mathrm{Cl}, 0.483-0.617 ; \mathrm{P}<0.001$, respectively) (Fig. 2). The difference was statistically significant in favor of the pial arterial filling scores dichotomized at $>2$ vs. $\leq 2$ compared with the pial arterial filling scores dichotomized at $>4$ vs. $\leq 4$ (DeLong's test, P-value $=0.006$ ). However, the difference was not statistically significant between the pial arterial filling scores dichotomized at $>3$ vs. $\leq 3$ compared with the pial arterial filling scores dichotomized at $>4$ vs. $\leq 4$ (DeLong's test, P-value $=0.274$ ) and between the pial arterial filling scores dichotomized at $>2$ vs. $\leq 2$ compared with the pial arterial filling scores dichotomized at $>3$ vs. $\leq 3$ (DeLong's

Table 2. Comparison of demographics and clinical and radiological parameters according to clinical outcome at 3 months after stroke

\begin{tabular}{|c|c|c|c|}
\hline Variable & $m R S>2(n=18)$ & $m R S \leq 2(n=20)$ & P-value \\
\hline \multicolumn{4}{|l|}{ Demographics } \\
\hline Age & $78.3 \pm 6.0$ & $67.1 \pm 10.4$ & $<0.001$ \\
\hline Male sex & $8(44.4)$ & $13(65.0)$ & 0.203 \\
\hline \multicolumn{4}{|l|}{ Risk factor } \\
\hline Diabetes mellitus & $6(33.3)$ & $5(25.0)$ & 0.724 \\
\hline Hypertension & $7(38.9)$ & $13(65.0)$ & 0.107 \\
\hline Atrial fibrillation & $13(72.2)$ & $11(55.0)$ & 0.328 \\
\hline Smoking & $8(44.4)$ & $4(20.0)$ & 0.164 \\
\hline Hyperlipidemia & $4(22.2)$ & $6(30.0)$ & 0.719 \\
\hline Previous stroke & $2(11.1)$ & $4(20.0)$ & \\
\hline Lesion location & & & 0.703 \\
\hline Middle cerebral artery & $11(61.1)$ & $11(55.0)$ & \\
\hline Internal carotid artery & $7(38.9)$ & $9(45.0)$ & \\
\hline Stroke subtype & & & 0.381 \\
\hline Large artery atherosclerosis & $4(22.2)$ & $4(20)$ & \\
\hline Cardioembolism & $13(72.2)$ & $11(55.0)$ & \\
\hline SOD & $0(10.0)$ & $2(10.0)$ & \\
\hline SUD & $1(5.6)$ & $3(15.0)$ & \\
\hline NIHSS score at admission & $17.7 \pm 4.3$ & $12.9 \pm 6.5$ & 0.011 \\
\hline Intravenous thrombolysis & $16(88.9)$ & $15(75.0)$ & 0.410 \\
\hline Onset to reperfusion time (minutes) & $189.1 \pm 51.3$ & $216.7 \pm 148.9$ & 0.444 \\
\hline Procedure time (minutes) & $50.1 \pm 36.4$ & $35.5 \pm 34.1$ & 0.209 \\
\hline $\mathrm{mTICl}(2 \mathrm{~B}$ or 3$)$ & $15(83.3)$ & $19(95.0)$ & 0.328 \\
\hline Pial arterial filling score & $2.4 \pm 1.2$ & $3.6 \pm 0.8$ & 0.002 \\
\hline Dichotomized score (>4 vs. $\leq 4$ ) & $0 / 18(0 / 100)$ & 2/18 (10.0/90.0) & 0.488 \\
\hline Dichotomized score (>3 vs. $\leq 3$ ) & $5 / 13(27.8 / 72.2)$ & $11 / 9(55.0 / 45.0)$ & 0.112 \\
\hline Dichotomized score (>2 vs. $\leq 2$ ) & $7 / 11(38.9 / 61.1)$ & $18 / 2(90.0 / 10.0)$ & 0.002 \\
\hline
\end{tabular}

Values are presented as mean \pm standard deviation or number (\%) unless otherwise indicated.

mRS, modified Rankin Scale; SOD, stroke of other determined etiology; SUD, stroke of undetermined etiology; NIHSS, National Institutes of Health Stroke Scale; $\mathrm{mTICl}$, modified treatment in cerebral infarction. 
Table 3. Association factors for favorable outcome at 3 months after stroke

\begin{tabular}{|c|c|c|c|c|}
\hline & OR $(95 \% \mathrm{Cl})$ & P-value & Adjusted* OR (95\% CI) & P-value \\
\hline \multicolumn{5}{|l|}{ Demographics } \\
\hline Age & $0.825(0.720-0.945)$ & 0.006 & & \\
\hline Male sex & $2.321(0.628-8.579)$ & 0.207 & & \\
\hline \multicolumn{5}{|l|}{ Risk factor } \\
\hline Diabetes mellitus & $0.667(0.163-2.727)$ & 0.573 & & \\
\hline Hypertension & $2.918(0.780-10.924)$ & 0.112 & & \\
\hline Atrial fibrillation & $0.470(0.121-1.825)$ & 0.275 & & \\
\hline Smoking & $0.313(0.074-1.315$ & 0.113 & & \\
\hline Hyperlipidemia & $1.500(0.346-6.498)$ & 0.588 & & \\
\hline Previous stroke & $2.000(0.320-12.510)$ & 0.459 & & \\
\hline NIHSS score at admission & $0.839(0.720-0.977)$ & 0.024 & & \\
\hline Intravenous thrombolysis & $0.375(0.063-2.234)$ & 0.281 & & \\
\hline Onset to reperfusion time (minutes) & $1.002(0.996-1.009)$ & 0.462 & & \\
\hline Procedure time (minutes) & $0.987(0.966-1.008)$ & 0.224 & & \\
\hline $\mathrm{mTICI}(2 \mathrm{~B}$ or 3$)$ & $3.800(0.358-40.336)$ & 0.268 & & \\
\hline Pial arterial filling score & $2.917(0.321-6.443)$ & 0.008 & $3.724(1.141-12.156)$ & 0.029 \\
\hline Dichotomized score (>3 vs. $\leq 3$ ) & $3.718(0.819-12.337)$ & 0.095 & $2.374(0.380-14.814)$ & 0.355 \\
\hline Dichotomized score (>2 vs. $\leq 2$ ) & $14.143(2.479-80.682)$ & 0.003 & $84.893(2.680-2689.571)$ & 0.012 \\
\hline
\end{tabular}

OR, odds ratio; $\mathrm{Cl}$, confidence interval; NIHSS, National Institutes of Health Stroke Scale; mTICl, modified treatment in cerebral infarction.

${ }^{*}$ Adjusted for age and NIHSS score at admission.

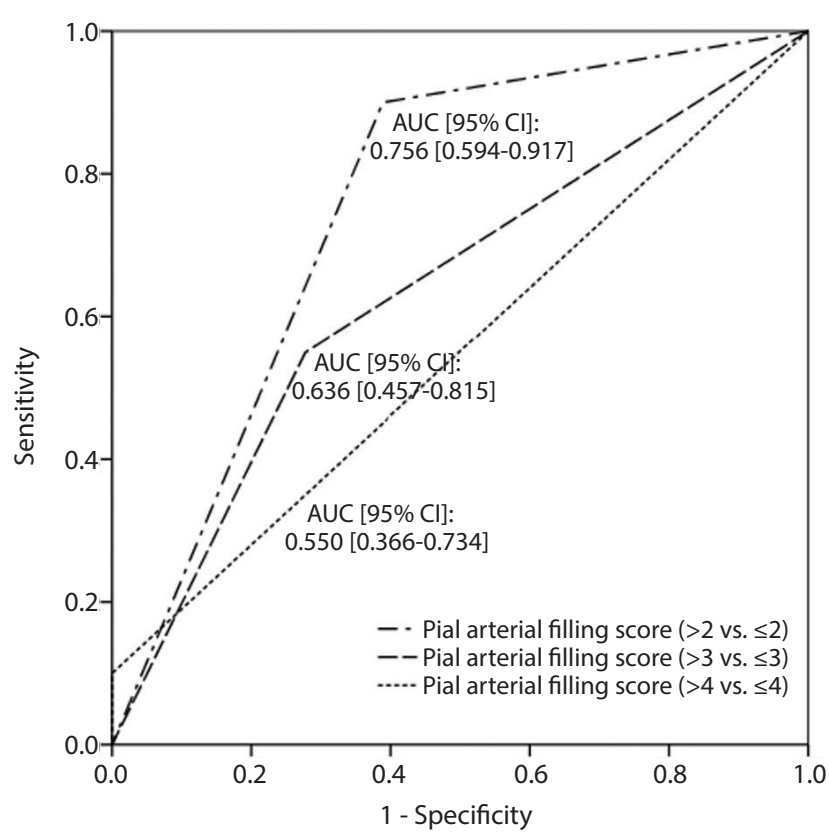

Fig. 2. Results of receiver operating characteristic analysis for investigating functional outcome using each dichotomized pial arterial filling score ( $>4$ vs. $\leq 4,>3$ vs. $\leq 3$, and $>2$ vs. $\leq 2$ ). AUC, area under the receiver-operating characteristic curve; $\mathrm{Cl}$, confidence interval. test, P-value $=0.073)$.

\section{DISCUSSION}

We found that pial arterial filling scores were higher in patients with favorable outcomes than in those with unfavorable outcomes. In particular, a pial arterial filling score cut-off of 2 on mpCTA was significantly associated with favorable clinical outcomes at 3 months after endovascular treatment for acute ischemic stroke with large vessel occlusion of the anterior circulation within 6 hours since last seen normal.

The appropriate pial arterial filling score cut-off for determining tissue salvageability and selecting suitable candidates for endovascular treatment has not been externally validated, although recommendations have been previously made. Specifically, the Endovascular Treatment for Small Core and Anterior Circulation Proximal Occlusion with Emphasis on Minimizing CT to Recanalization Times (ESCAPE) trial demonstrated that moderate to good collateral circulation leads to decreased mortality and significantly improved clinical outcome after endovascular treatment. ${ }^{14}$ For decision 
making with mpCTA, the ESCAPE trial considered a pial arterial filling score of $>3$ to indicate the presence of salvageable brain; thus, scores of $0-3$ were considered exclusion criteria for endovascular treatment. ${ }^{14}$ Furthermore, in the Interventional Management of Stroke trial, the rate of favorable outcomes in patients with poor collaterals treated with endovascular therapy ranged from only $5.3 \%$ to $20.6 \%$ when they were assessed using three different ordinal scoring systems. ${ }^{15}$ Although criteria for candidate of endovascular treatment in our institution did not include degree of pial collateral circulation, our external validation of the appropriate cut-off score for determining collateral status using $\mathrm{mpC}-$ TA within 6 hours since last seen normal suggests that a cutoff score of 2 is more suitable than that of 3 or 4 . The reason might be because our study, which included patients with a proximal intracranial occlusion in the anterior circulation that presented within 6 hours after symptom onset, differs from the ESCAPE trial, and because patients with a collateral score of 3 have sufficient collateral perfusion and reversible brain tissues and may thus be good candidates for endovascular revascularization within 6 hours since last seen normal. Practically, as a noninvasive collateral assessment tool for the effectiveness of endovascular treatment in acute ischemic stroke with large vessel occlusion, application of this newly identified pial collateral score cut-off may result in favorable clinical outcomes.

Despite these insights, there are limitations to our study that need to be taken into consideration when interpreting our data. The present study was a single-center study with a limited number of patients. Furthermore, a previous report showed better interrater reliability to assess pial arterial filling scores than the present study. However, for a reduced time interval from within 12 hours since last seen normal to within 6 hours since last seen normal, a smaller number of patients can be expected. Therefore, further studies with larger patient cohorts need to be conducted. Additionally, the timing of the CTA contrast injection relative to imaging is likely to be critical. ${ }^{16}$ If CTA images are acquired too early, different phases may fail to be measured, leading to overestimation of the pial arterial filling score. As such, poor cardiac function can also interfere with pial arterial filling, as shown on mpCTA.

\section{CONCLUSION}

Pial arterial filling scores dichotomized at $\leq 2$ vs. $>2$ as deter- mined by mpCTA for a 6-hour time window appear to be suitable for predicting clinical outcome at 3 months after mechanical thrombectomy for acute ischemic stroke with large vessel occlusion of the anterior circulation. Also, critical re-appraisal for the cut-off score of mpCTA should be re-assessed by well-designed further trials.

\section{REFERENCES}

1. Goyal M, Menon BK, van Zwam WH, Dippel DW, Mitchell PJ, Demchuk AM, et al. Endovascular thrombectomy after large-vessel ischaemic stroke: a meta-analysis of individual patient data from five randomised trials. Lancet 2016;387:17231731

2. Hong KS, Ko SB, Yu KH, Jung C, Park SQ, Kim BM, et al. Update of the korean clinical practice guidelines for endovascular recanalization therapy in patients with acute ischemic stroke. J Stroke 2016;18:102-113

3. Powers WJ, Derdeyn CP, Biller J, Coffey CS, Hoh BL, Jauch EC, et al. 2015 American Heart Association/American Stroke Association focused update of the 2013 guidelines for the early management of patients with acute ischemic stroke regarding endovascular treatment: a guideline for healthcare professionals from the American Heart Association/American Stroke Association. Stroke 2015;46:3020-3035

4. Liebeskind DS. Collateral lessons from recent acute ischemic stroke trials. Neurol Res 2014;36:397-402

5. Christoforidis GA, Karakasis C, Mohammad Y, Caragine LP, Yang M, Slivka AP. Predictors of hemorrhage following intra-arterial thrombolysis for acute ischemic stroke: the role of pial collateral formation. AJNR Am J Neuroradiol 2009;30:165-170

6. Bang OY, Saver JL, Kim SJ, Kim GM, Chung CS, Ovbiagele B, et al. Collateral flow predicts response to endovascular therapy for acute ischemic stroke. Stroke 2011;42:693-699

7. Christoforidis GA, Mohammad Y, Kehagias D, Avutu B, Slivka AP. Angiographic assessment of pial collaterals as a prognostic indicator following intra-arterial thrombolysis for acute ischemic stroke. AJNR Am J Neuroradiol 2005;26:1789-1797

8. van den Wijngaard IR, Boiten J, Holswilder G, Algra A, Dippel DW, Velthuis BK, et al. Impact of collateral status evaluated by dynamic computed tomographic angiography on clinical outcome in patients with ischemic stroke. Stroke 2015;46:33983404

9. Menon BK, d'Esterre CD, Qazi EM, Almekhlafi M, Hahn L, Demchuk AM, et al. Multiphase ct angiography: a new tool for the 
imaging triage of patients with acute ischemic stroke. Radiology 2015;275:510-520

10. Yu AY, Zerna C, Assis Z, Holodinsky JK, Randhawa PA, Najm M, et al. Multiphase ct angiography increases detection of anterior circulation intracranial occlusion. Neurology 2016;87:609-616

11. Yang CY, Chen YF, Lee CW, Huang A, Shen Y, Wei C, et al. Multiphase ct angiography versus single-phase CT angiography: comparison of image quality and radiation dose. AJNR Am J Neuroradio/ 2008;29:1288-1295

12. Demchuk AM, Menon BK, Goyal M. Comparing vessel imaging: noncontrast computed tomography/computed tomographic angiography should be the new minimum standard in acute disabling stroke. Stroke 2016;47:273-281

13. Yoo AJ, Simonsen CZ, Prabhakaran S, Chaudhry ZA, Issa MA, Fugate JE, et al. Refining angiographic biomarkers of revascu- larization: improving outcome prediction after intra-arterial therapy. Stroke 2013:44:2509-2512

14. Goyal M, Demchuk AM, Menon BK, Eesa M, Rempel JL, Thornton J, et al. Randomized assessment of rapid endovascular treatment of ischemic stroke. N Engl J Med 2015;372:1019-1030

15. Menon BK, Qazi E, Nambiar V, Foster LD, Yeatts SD, Liebeskind D, et al. Differential effect of baseline computed tomographic angiography collaterals on clinical outcome in patients enrolled in the interventional management of stroke iii trial. Stroke 2015;46:1239-1244

16. Mortimer AM, Simpson E, Bradley MD, Renowden SA. Computed tomography angiography in hyperacute ischemic stroke: prognostic implications and role in decision-making. Stroke 2013:44:1480-1488 